\title{
SUPER EXPONENTIALLY CONVERGENT APPROXIMATION TO THE SOLUTION OF THE SCHRÖDINGER EQUATION IN ABSTRACT SETTING
}

\author{
I.P. GAVRILYUK ${ }^{1}$
}

\begin{abstract}
We have developed an approximation to the solution of the Schrödinger equation in abstract setting. The accuracy of our approximation depends on the smoothness of this solution. We show that for the analytical initial vectors our approximation possesses a super exponential convergence rate.
\end{abstract}

2000 Mathematics Subject Classification: 65F50, 65F30, 46B28, 47A80.

Keywords: exact representation of the solution, super exponentially convergent algorithms, Cayley transform, Schrödinger equation.

\section{Introduction}

In the theory of operators in Hilbert space the Cayley transform $T_{\gamma, \delta}^{\alpha, \beta}=(\alpha I+\beta A)(\gamma I-$ $\delta A)^{-1}, \alpha, \beta, \gamma, \delta \in \mathbb{C}$ is frequently used to switch from the study of the closed but in general unbounded linear operator $A$ with a dense domain $D(A)(\bar{D}(A)=H)$ to that of bounded operators $T_{\gamma, \delta}^{\alpha, \beta}$. In the classical case $T_{\gamma}=(\gamma I+A)(\gamma I-A)^{-1}, \gamma=-i$, this transform converts the self-adjoint (symmetric, dissipative) operators A into unitary (respectively, isometric, contractive) operators $T_{\gamma}$ (see [1]).

The Cayley transform can also be used to turn the processes with a continuous time parameter into such with discrete time (see, e.g., [20] dealing with the prediction theory of stationary stochastic processes).

In $[2,5-7,11-14,16,17]$, the Cayley transform was used to obtain explicit and constructive representations of the solutions of various evolution differential equations with operator coefficients, where, in fact, the solutions with a continuous time parameter were represented in terms of those with discrete time. Moreover, these representations can be considered as a method for separating time and "spatial" variables. A further important feature of these representation is the fact that they serve as the basis for algorithms without accuracy saturation, i.e., their accuracy increases automatically and unboundedly with increasing smoothness of the solution.

In the present paper, we derive an explicit representation of the solution operator (and, therefore of the solution as well) of the Schrödinger differential equation in abstract setting, i.e., with the operator coefficient in some Hilbert space. We propose an approximation with

\footnotetext{
${ }^{1}$ University of Cooperative Education, Staatliche Studienakademie Thueringen, Berufsakademie Eisenach, Am Wartenberg 2, 99817 Eisenach, Germany. E-mail: ipg@ba-eisenach.de
} 
an accuracy depending on the smoothness of this solution. We show that for analytical initial vectors our approximation possesses a super exponential convergence rate. Note that the exponential convergence is needed to provide algorithms of the optimal or near optimal complexity [7-9]. In [7-9,14,15], a representation through an improper Dunford-Cauchy integral along the path enveloping the spectrum of operator coefficient and the Sinc-quadrature were proposed to obtain exponentially convergent approximations.

The paper is organized as follows. In Section 2, we derive an explicit representation of the solution operator as a series where the time variable is separated in Laguerre polynomials and the "spatial" operator in the powers of a Cayley transform. The convergence properties of the series are studied. Section 3 is devoted to the truncated series as an approximation to the exact solution. We show that this approximation features no accuracy saturation, i.e., its accuracy depends on the smoothness of the exact solution. In the case of an analytical initial vector, we prove a super exponential convergence of our approximation. In Section 4, we derive another form of representation for the exact solution and a modified approximation (truncated series with $N$ terms) to this solution. The error of the new approximation tends to zero as $N \rightarrow \infty$ and as $t \rightarrow 0$. The second property allows us to develop a preconditioning technique which is the topic of Section 5.

\section{Exact representation of the solution}

Let $H$ be a complex Hilbert space and $B$ - a self-adjoint unbounded positive definite operator in this space with the domain $D(B)$ and the spectrum $\Sigma(B) \in\left[\lambda_{0}, \infty\right), \lambda_{0}>0$. Let us consider the following initial value problem for the Schrödinger equation:

$$
\dot{x}(t)=i B x(t), \quad x(0)=x_{0} .
$$

The solution operator of this problem is the operator exponential family $S(t)=e^{i B t}$ such that the solution of the IVP is given by $x(t)=S(t) x_{0}$. Using the well-known expansion [3, v.2, Ch. 10.12]

$$
(1-z)^{-\alpha-1} e^{\frac{t z}{z-1}}=\sum_{n=0}^{\infty} L_{n}^{(\alpha)}(t) z^{n}
$$

after the substitution $z \rightarrow i B(i B-I)^{-1}$ we obtain formally

$$
e^{i B t}=-(i B-I)^{-1} \sum_{n=0}^{\infty} L_{n}^{(0)}(t) T^{n},
$$

where $T=T(B)=i B(i B-I)^{-1}$ is the (non-classical) Cayley transform of the operator $B$.

Now, the solution of the IVP can be formally represented by

$$
x(t)=\sum_{n=0}^{\infty} L_{n}^{(0)}(t) u_{n},
$$

where the sequence $\left\{u_{n}\right\}$ is defined by the recursion

$$
\begin{aligned}
u_{0} & =-(i B-I)^{-1} x_{0}, \\
u_{p+1} & =i B(i B-I)^{-1} u_{p}, \quad p=0,1, \ldots
\end{aligned}
$$

In other words, the elements $u_{p}$ of the sequence are solutions of the operator equations

$$
\begin{aligned}
(i B-I) u_{0} & =-x_{0}, \\
(i B-I) u_{p+1} & =i B u_{p}, \quad p=0,1, \ldots
\end{aligned}
$$


By formal differentiation and using the well-known relations $\frac{d}{d t} L_{n}^{(\alpha)}(t)=-L_{n+1}^{(\alpha+1)}(t)$, $L_{p-1}^{(1)}(t)=\sum_{\nu=0}^{p-1} L_{\nu}^{(0)}(t)$ we obtain from $(2.4)$ the series

$$
x_{1}(t)=-\sum_{p=1}^{\infty} \sum_{\nu=0}^{p-1} L_{\nu}^{(0)}(t) u_{p} .
$$

The next Lemma resolves the convergence conditions for series (2.4), (2.7).

Lemma 2.1. Let $x_{0} \in D\left(B^{\sigma}\right)$, then

1) series (2.4) converges in $H$ uniformly in $t$ on an arbitrary finite closed interval $[0, b]$ (on an arbitrary finite closed interval $[a, b], 0<a<b$ ) and $x(t)$ is a continuous function on $[0, b]$ (on $[a, b])$ provided that $\sigma>2(\sigma>3 / 2)$,

2) series (2.7) converges in $H$ uniformly in $t \in[0, b]$ (on $[a, b]), x_{1}(t)$ is a continuous function on $[0, b](($ on $[a, b]))$ and $\dot{x}(t)=x_{1}(t)$ provided that $\sigma>4(\sigma>5 / 2)$,

3) $x(t) \in D(B) \forall t \in[0, b]$ (on $[a, b])$ provided that $\sigma>4(\sigma>5 / 2)$.

Proof. We can represent

$$
\begin{aligned}
u_{p+1} & =\left[i B(i B-I)^{-1}\right]^{p} u_{0}=\int_{\lambda_{0}}^{\infty}\left(\frac{i \lambda}{i \lambda-1}\right)^{p} d E_{\lambda} x_{0} \\
& =\int_{\lambda_{0}}^{\infty}\left(\frac{\lambda}{\lambda+i}\right)^{p} d E_{\lambda} x_{0}=\int_{\lambda_{0}}^{\infty} \frac{1}{\lambda^{\sigma}}\left(\frac{\lambda^{2}-i \lambda}{1+\lambda^{2}}\right)^{p} d E_{\lambda} x_{0}^{\sigma} \\
& =\int_{\lambda_{0}}^{\infty} \frac{1}{\lambda^{\sigma}}\left(\frac{\lambda^{p-\sigma}}{\left(1+\lambda^{2}\right)^{p / 2}}\right)^{p} e^{-i p \varphi} d E_{\lambda} x_{0}^{\sigma}
\end{aligned}
$$

where $x_{0}^{\sigma}=B^{\sigma} x_{0}, \varphi=\arccos r, r=\lambda / \sqrt{\lambda^{2}+1}$. This yields the estimate

$$
\left\|u_{p+1}\right\| \leqslant \sup _{\lambda \in\left[\lambda_{0}, \infty\right)} \Phi(\lambda)\left\|x_{0}^{\sigma}\right\|,
$$

where $\Phi(\lambda)=\frac{\lambda^{p-\sigma}}{\left(1+\lambda^{2}\right)^{p / 2}}=\theta^{\sigma / 2} \frac{1}{(1+\theta)^{p / 2}}=\Psi(\theta), \theta=\lambda^{-2}$ and $E_{\lambda}$ is the spectral family of $B$. From $\Psi^{\prime}(\theta)=\theta^{\sigma / 2-1}(1+\theta)^{-p / 2-1}\left[\frac{\sigma}{2}(1+\theta)-\frac{p}{2} \theta\right]$ we obtain the extremum point $\theta=\frac{\sigma}{p-\sigma}$ and the maximum value

$$
\Phi_{\max }=\Phi\left(\frac{\sigma}{p-\sigma}\right) \leqslant c(\sigma) p^{-\sigma / 2}
$$

with some constant $c=c(\sigma)$ independent of $p$. Now estimate (2.9) takes the form

$$
\left\|u_{p+1}\right\| \leqslant c p^{-\sigma / 2}\left\|x_{0}^{\sigma}\right\| .
$$

Let us recall the following properties of Laguerre polynomials (see, e.g., [21, p. 243, 248] and [3, vol. 2, Ch. 10.18]):

$$
\begin{aligned}
& L_{n}^{(\alpha)}(t)=\pi^{-1 / 2} e^{t / 2} t^{-\alpha / 2-1 / 4} n^{\alpha / 2-1 / 4}\left[\cos (2 \sqrt{n t}-\beta \pi)+\mathcal{O}\left(n^{-1 / 2}\right)\right], t \in[a, b], \\
& 0<a<b<\infty, \beta=(2 \alpha+1) / 4, \alpha>-1, \\
& \left|L_{n}^{(\alpha)}(t)\right| \leqslant c n^{\frac{\alpha}{2}-\frac{1}{4}} t^{-\frac{\alpha}{2}-\frac{1}{4}} e^{\frac{t}{2}}\left(1+n^{-\frac{1}{4}} t^{\frac{5}{4}}\right), \alpha+\frac{1}{2} \geqslant 0, t \geqslant 0, \\
& e^{-t / 2}\left|L_{n}^{(0)}\right| \leqslant 1, t \geqslant 0,
\end{aligned}
$$


where $c$ is a constant independent of $k$ and $0<a<b$ arbitrary fixed numbers. Therefore, series (2.4), (2.7) are majorized by the number series

$$
c \begin{cases}t^{-\frac{1}{4}} e^{\frac{t}{2}} \sum_{p=1}^{\infty} p^{-\sigma / 2-1 / 4}, & \text { if } t \in[a, b] \\ e^{\frac{t}{2}} \sum_{p=1}^{\infty} p^{-\sigma / 2}, & \text { if } t \in[0, b]\end{cases}
$$

and

$$
c \begin{cases}t^{-\frac{1}{4}} e^{\frac{t}{2}} \sum_{p=1}^{\infty} p^{-\sigma / 2+1 / 4}, & \text { if } t \in[a, b] \\ e^{\frac{t}{2}} \sum_{p=1}^{\infty} p^{-\sigma / 2+1}, & \text { if } t \in[0, b]\end{cases}
$$

respectively which converge uniformly in $t$ provided that

$$
\left\{\begin{array} { l l } 
{ \sigma > 3 / 2 , } & { \text { if } t \in [ a , b ] } \\
{ \sigma > 2 , } & { \text { if } t \in [ 0 , b ] }
\end{array} \text { and } \left\{\begin{array}{ll}
\sigma>5 / 2, & \text { if } t \in[a, b] \\
\sigma>4, & \text { if } t \in[0, b]
\end{array}\right.\right.
$$

Let us prove that series (2.4) satisfies the differential equation

$$
\begin{aligned}
\dot{x} & (t)-i B x(t)=-\sum_{p=1}^{\infty} \sum_{\nu=0}^{p-1} L_{\nu}^{(0)}(t) \int_{\lambda_{0}}^{\infty}\left(\frac{\lambda}{\lambda+i}\right)^{p-1} d E_{\lambda} x_{0}-i \sum_{p=0}^{\infty} L_{p}^{(0)}(t) \int_{\lambda_{0}}^{\infty} \lambda\left(\frac{\lambda}{\lambda+i}\right)^{p-1} d E_{\lambda} x_{0} \\
= & -\sum_{p=1}^{\infty} \sum_{\nu=0}^{p-1} L_{\nu}^{(0)}(t) \int_{\lambda_{0}}^{\infty}\left[\sum_{\mu=p}^{\infty}\left(\frac{\lambda}{\lambda+i}\right)^{\mu-1}-\sum_{\mu=p+1}^{\infty}\left(\frac{\lambda}{\lambda+i}\right)^{\mu-1}\right] d E_{\lambda} x_{0} \\
- & i \sum_{p=0}^{\infty} L_{p}^{(0)}(t) \int_{\lambda_{0}}^{\infty} \lambda\left(\frac{\lambda}{\lambda+i}\right)^{p-1} d E_{\lambda} x_{0} \\
= & -\sum_{p=1}^{\infty} \sum_{\nu=0}^{p-1} L_{\nu}^{(0)}(t) \int_{\lambda_{0}}^{\infty} \sum_{\mu=p}^{\infty}\left(\frac{\lambda}{\lambda+i}\right)^{\mu-1} d E_{\lambda} x_{0}+\sum_{p=2}^{\infty} \sum_{\nu=0}^{p-2} L_{\nu}^{(0)}(t) \int_{\lambda_{0}}^{\infty} \sum_{\mu=p}^{\infty}\left(\frac{\lambda}{\lambda+i}\right)^{\mu-1} d E_{\lambda} x_{0} \\
- & i \sum_{p=0}^{\infty} L_{p}^{(0)}(t) \int_{\lambda_{0}}^{\infty} \lambda\left(\frac{\lambda}{\lambda+i}\right)^{p-1} d E_{\lambda} x_{0} \\
= & -\sum_{p=1}^{\infty} L_{p-1}^{(0)}(t) \int_{\lambda_{0}}^{\infty} \sum_{\mu=p}^{\infty}\left(\frac{\lambda}{\lambda+i}\right)^{\mu-1} d E_{\lambda} x_{0}-i \sum_{p=0}^{\infty} L_{p}^{(0)}(t) \int_{\lambda_{0}}^{\infty} \lambda\left(\frac{\lambda}{\lambda+i}\right)^{p-1} d E_{\lambda} x_{0} \\
= & -\sum_{p=0}^{\infty} L_{p}^{(0)}(t) \int_{\lambda_{0}}^{\infty}\left[\sum_{\mu=p+1}^{\infty}\left(\frac{\lambda}{\lambda+i}\right)^{\mu-1} d+i \lambda\left(\frac{\lambda}{\lambda+i}\right)^{p-1} d E_{\lambda} x_{0}\right. \\
= & -\sum_{p=0}^{\infty} L_{p}^{(0)}(t) \int_{\lambda_{0}}^{\infty}\left[\left(\frac{\lambda}{\lambda+i}\right)^{p} \frac{\lambda+i}{i}+i \lambda\left(\frac{\lambda}{\lambda+i}\right)^{p-1}\right] d E_{\lambda} x_{0} \\
= & -\sum_{p=0}^{\infty} L_{p}^{(0)}(t) \int_{\lambda_{0}}^{\infty}\left(\frac{\lambda}{\lambda+i}\right)^{p-1}\left[\frac{\lambda}{i}+i \lambda\right] d E_{\lambda} x_{0}=0 . \\
& =0.16)
\end{aligned}
$$




\section{Approximation I}

We consider the truncated series

$$
x_{N}(t)=\sum_{n=0}^{N} L_{n}^{(0)}(t) u_{n},
$$

as an approximation to the exact solution. The next theorem characterizes the goodness of this approximation.

Theorem 3.1. Let $x_{0} \in D\left(B^{\sigma}\right)$, then the following estimates hold true

$$
\left\|x(t)-x_{N}(t)\right\| \leqslant c\left\|B^{\sigma} x_{0}\right\|\left\{\begin{array}{ll}
t^{-\frac{1}{4}} e^{\frac{t}{2}} N^{-\sigma / 2+5 / 4}, & \text { if } t \in[a, b] \\
e^{\frac{t}{2}} N^{-\sigma / 2+2}, & \text { if } t \in[0, b]
\end{array},\right.
$$

with some constant $c$ independent of $N$.

Proof. We have

$$
\begin{aligned}
\left\|x(t)-x_{N}(t)\right\| \leqslant c\left\|B^{\sigma} x_{0}\right\| \begin{cases}t^{-\frac{1}{4}} e^{\frac{t}{2}} \sum_{p=N+1}^{\infty} p^{-\sigma / 2+1 / 4}, & \text { if } t \in[a, b] \\
e^{\frac{t}{2}} \sum_{p=N+1}^{\infty} p^{-\sigma / 2+1}, & \text { if } t \in[0, b]\end{cases} \\
\leqslant c\left\|B^{\sigma} x_{0}\right\| \begin{cases}t^{-\frac{1}{4}} e^{\frac{t}{2}} N^{-\sigma / 2+5 / 4}, & \text { if } t \in[a, b] \\
e^{\frac{t}{2}} N^{-\sigma / 2+2}, & \text { if } t \in[0, b]\end{cases}
\end{aligned}
$$

Let us recall that vectors from $\bigcap_{n=1}^{\infty} D\left(B^{n}\right)$ are called $C^{\infty}$-vectors for the operator $B[18$, Ch. 1, 99.20]. For example, for the operator $B=\frac{d}{d x}$ the functions of the class $C^{\infty}$ are $C^{\infty}$-vectors. A vector $f$ is called analytical for $B$ if $f \in C^{\infty}$ and the power series

$$
\sum_{n=0}^{\infty} \frac{t^{n}}{n !}\left\|B^{n} f\right\|
$$

possesses a positive convergence radius.

Example 3.1. Let $B$ be an operator in $\mathbb{R}^{n}$ represented by a matrix $B$. Each vector $f \in \mathbb{R}^{n}$ is analytical because the power series

$$
\sum_{n=0}^{\infty} \frac{t^{n}}{n !}\left\|B^{n} f\right\|
$$

converges for all $t \in \mathbb{R}$ due to $\left\|B^{n} f\right\| \leqslant\|B\|^{n}\|f\|$.

Example 3.2. Let us consider the operator in $L_{2}(0, \pi)$ defined by

$$
\begin{aligned}
D(B) & =\left\{u \in H^{2}(0, \pi): u(0)=u(\pi)=0\right\}, \\
B & =-\frac{d^{2} u}{d t^{2}} \quad \forall u \in D(B) .
\end{aligned}
$$


The vector $f=f(t)=\sin t$ is analytical for $B$ since $B^{n} f=(-1)^{n+1} \sin t,\left\|B^{n} f\right\|=$ $\left(\int_{0}^{\pi} \sin ^{2} t d t\right)^{1 / 2}=\sqrt{\pi / 2} \forall n$ and the power series

$$
\sum_{n=0}^{\infty} \frac{t^{n}}{n !}\left\|B^{n} f\right\|=\sqrt{\pi / 2} \sum_{n=0}^{\infty} \frac{t^{n}}{n !}=\sqrt{\pi / 2} e^{t}
$$

converges for all $t \in \mathbb{R}$.

Example 3.3. Let us consider the operator in $L_{2}(-\infty, \infty)$ defined by

$$
\begin{aligned}
D(B) & =\left\{u \in H^{2}(-\infty, \infty): \psi(-\infty)=\psi(\infty)=0\right\}, \\
B & =-\frac{\hbar^{2}}{2 m} \frac{d^{2} \psi}{d x^{2}}+\frac{m \omega^{2}}{2} x^{2} \psi \quad \forall \psi \in D(B) .
\end{aligned}
$$

In the quantum mechanics, the operator $B$ is a Hamiltonian of an oscillated particle of mass $m$ subject to a potential $V(x)$ given by $V(x)=\frac{1}{2} m \omega^{2} x^{2}$, where $\omega$ is the angular frequency of the oscillator. It can be shown that the normalized eigenfunctions (subject to $\left.\int_{-\infty}^{\infty} \psi^{2}(x) d x=1\right)$ are

$$
\psi_{n}(x)=C_{n} e^{-\xi^{2} / 2} H_{n}(\xi)
$$

where $x=\alpha \xi, \alpha=\sqrt{\frac{\hbar}{m \omega}}, C_{n}=\frac{1}{\sqrt{\alpha}} \cdot \frac{1}{\sqrt{2^{n} n ! \sqrt{\pi}}}$ and $H_{n}(\xi)$ are Hermit polynomials [21, 22]. These eigenfunctions correspond to the eigenvalues $E_{n}=(n+1 / 2) \hbar \omega$. The vector $u_{0}(x)=$ $\sum_{k=0}^{m} \gamma_{k} \psi_{k}(x)$ with a fixed $m$ is analytical for $B$ since

$$
\begin{aligned}
B^{n} u_{0} & =(\hbar \omega)^{n} \sum_{k=0}^{m} \gamma_{k}(k+1 / 2)^{n} \psi_{k}(x) \\
\left\|B^{n} u_{0}\right\| & =(\hbar \omega)^{n}\left(\int_{-\infty}^{\infty}\left[\sum_{k=0}^{m} \gamma_{k}(k+1 / 2)^{n} \psi_{k}(x)\right]^{2}\right)^{1 / 2} \\
& \leqslant(\hbar \omega)^{n} \sqrt{m}\left(\sum_{k=0}^{m} \gamma_{k}^{2}(k+1 / 2)^{2 n}\right)^{1 / 2}\left(\sum_{k=0}^{m} \int_{-\infty}^{\infty} \psi_{k}^{2}(x) d x\right)^{1 / 2} \\
& \leqslant(\hbar \omega)^{n} m(m+1 / 2)^{n}\left(\sum_{k=0}^{m} \gamma_{k}^{2}\right)^{1 / 2}
\end{aligned}
$$

and the power series

$$
\sum_{n=0}^{\infty} \frac{t^{n}}{n !}\left\|B^{n} f\right\| \leqslant m\left(\sum_{k=0}^{m} \gamma_{k}^{2}\right)^{1 / 2} \sum_{n=0}^{\infty} \frac{(\hbar \omega)^{n}(m+1 / 2)^{n}}{n !} t^{n}
$$

converges for all $t \in \mathbb{R}$.

The next theorem shows a super exponential convergence of approximation (3.1) provided that the initial vector is analytical. 
Theorem 3.2. If $x_{0}$ is analytical for $B$, then approximation (3.1) converges superexponentially with the error estimate

$$
\left\|x(t)-x_{N}(t)\right\| \leqslant c t^{-\frac{1}{4}} e^{\frac{t}{2}} e^{-\left(c_{1}-\varepsilon\right) N^{1 / 3}}\left\|\mid x_{0}\right\| \| \quad \forall \quad t \in[a, b], 0<a<b,
$$

where $c, c_{1}, a, b, \varepsilon \in\left(0, c_{1}\right)$ are positive constants independent of $N,\|\| x_{0}\left\|\mid=\sum_{k=0}^{\infty} \frac{r^{k}}{k !}\right\| B^{k} x_{0} \|$ and $r$ is the convergence radius of the power series $\sum_{k=0}^{\infty} \frac{s^{k}}{k !}\left\|B^{k} x_{0}\right\|$.

Proof. First of all we note that estimates (2.12) yield for $t \in[a, b]$

$$
\left\|x(t)-x_{N}(t)\right\| \leqslant c t^{-\frac{1}{4}} e^{\frac{t}{2}} \sum_{p=N+1}^{\infty} p^{-1 / 4}\left\|u_{p}\right\|,
$$

where

$$
\begin{aligned}
u_{p} & =\int_{\lambda_{0}}^{\infty} \frac{\lambda^{p-1}}{\left(1+\lambda^{2}\right)^{(p-1) / 2}} e^{-i(p-1) \varphi} d E_{\lambda} x_{0} \\
& =\int_{\lambda_{0}}^{\infty} e^{-\lambda s} \frac{\lambda^{p-1}}{\left(1+\lambda^{2}\right)^{(p-1) / 2}} e^{-i(p-1) \varphi}\left(\sum_{k=0}^{\infty} \frac{\lambda^{k} s^{k}}{k !}\right) d E_{\lambda} x_{0} \\
& =\int_{\lambda_{0}}^{\infty} \Phi_{s}(\lambda)\left(\sum_{k=0}^{\infty} \frac{\lambda^{k} s^{k}}{k !}\right) d E_{\lambda} x_{0} \\
& =\int_{\lambda_{0}}^{\infty} \Phi_{s}(\lambda) d E_{\lambda}\left(\sum_{k=0}^{\infty} \frac{s^{k}}{k !} B^{k} x_{0}\right)
\end{aligned}
$$

and

$$
\Phi_{s}(\lambda)=e^{-\lambda s} \frac{\lambda^{p-1}}{\left(1+\lambda^{2}\right)^{(p-1) / 2}} e^{-i(p-1) \varphi}=e^{-\lambda s-i(p-1) \varphi} \frac{1}{\left(1+\lambda^{-2}\right)^{(p-1) / 2}} .
$$

From the last equality we obtain the estimate

$$
\left\|u_{p}\right\| \leqslant \Phi_{\max }\left\|\left|x_{0} \|\right|\right.
$$

with $\Phi_{\max }=\sup _{\lambda \in\left[\lambda_{0}, \infty\right)} \Phi_{r}(\lambda)$. The extremum points of the function $\Phi_{r}(\lambda)$ are the solutions of the equation

$$
\begin{aligned}
\frac{d}{d \lambda}\left|\Phi_{r}(\lambda)\right|= & -r e^{-\lambda r} \frac{1}{\left(1+\lambda^{-2}\right)^{(p-1) / 2}}+\frac{p-1}{2} e^{-\lambda r} \frac{2 / \lambda^{3}}{\left(1+\lambda^{-2}\right)^{(p-1) / 2+1}} \\
& =-e^{-\lambda r} \frac{1}{\left(1+\lambda^{-2}\right)^{(p-1) / 2+1}}\left[r\left(1+\lambda^{-2}\right)-\frac{p-1}{\lambda^{3}}\right] \\
& =-e^{-\lambda r} \frac{1}{\lambda^{3}\left(1+\lambda^{-2}\right)^{(p-1) / 2+1}}\left[r \lambda^{3}+r \lambda-(p-1)\right]=0,
\end{aligned}
$$

i.e.,

$$
\lambda^{3}+\lambda-\frac{p-1}{r}=0
$$


Since the coefficient in the front of $\lambda$ is positive the cubic equation (3.13) possesses a positive real solution [4] $\lambda_{1}=-2 r_{*} \sinh \phi / 3$, where $r_{*}=\operatorname{sign}((p-1) / r) / \sqrt{3}<0$ and $\phi$ is defined by $\sinh \phi=-\frac{p-1}{2 r r_{*}^{3}}>0$, i.e. $\lambda_{1} \asymp \sinh \phi / 3 \asymp \sqrt[3]{p}$ (see e.g. [4, p.132]). Therefore, we have that $\Phi_{\max } \leqslant c e^{-c_{1} p^{1 / 3}}$ with some positive constants $c, c_{1}$ independent of $p$. This yields

$$
\begin{aligned}
& \left\|x(t)-x_{N}(t)\right\| \leqslant c t^{-\frac{1}{4}} e^{\frac{t}{2}} \sum_{p=N+1}^{\infty} p^{-1 / 4} e^{-c_{1} p^{1 / 3}}\left\|\left|x_{0} \|\right|\right. \\
& \leqslant c t^{-\frac{1}{4}} e^{\frac{t}{2}}\left\|\left|x _ { 0 } \left\|\left|\int_{N+1}^{\infty} x^{-1 / 4} e^{-c_{1} x^{1 / 3}} d x \leqslant c t^{-\frac{1}{4}} e^{\frac{t}{2}}\left\|\left|x_{0} \|\right| \int_{N+1}^{\infty}\left[x^{5 / 12} e^{-\varepsilon x^{1 / 3}}\right]\left[x^{-2 / 3} e^{-\left(c_{1}-\varepsilon\right) x^{1 / 3}}\right] d x\right.\right.\right.\right.\right. \\
& \leqslant c t^{-\frac{1}{4}} e^{\frac{t}{2}}\left\|\left|x _ { 0 } \left\|\left|\int_{N+1}^{\infty} e^{-\left(c_{1}-\varepsilon\right) x^{1 / 3}} d\left(x^{1 / 3}\right) \leqslant c t^{-\frac{1}{4}} e^{\frac{t}{2}}\left\|\mid x_{0}\right\| e^{-\left(c_{1}-\varepsilon\right) N^{1 / 3}}\right.\right.\right.\right.
\end{aligned}
$$

with some constants $c, c_{1}, 0<\varepsilon<c_{1}$ independent of $N$.

Remark 3.1. For a fixed $n \times n$-matrix $B$ considered as an operator in $\mathbb{R}^{n}$, an arbitrary vector $x \in \mathbb{R}^{n}$ is analytical. If a matrix $B=B_{h}$ is a grid approximation of an unbounded operator $B$, then $n$, as well as the norm of $B$, tend to infinity as the characteristic mesh value $h$ tends to zero. We call such operators quasi-bounded. In this case, the convergence radius of the series

$$
\sum_{n=0}^{\infty} \frac{\left\|B_{h}^{n} x_{0}\right\| t^{n}}{n !}
$$

for the initial vector $x_{0}$ can tend to zero together with $h \rightarrow 0$ and the above analyticity definition has no more sense. This fact motivates the following definition of the analytical vectors for $B_{h}$.

Definition 3.1. A vector $x_{0} \in \mathbb{R}^{n}$ is called analytical for $B=B_{h}$ uniformly in $h$ if series (3.15) possesses a positive convergence radius independent of $h$.

Example 3.4. Let $\omega=\omega_{h}=\left\{\xi_{i}=i h: i=1,2, \ldots, n ; h=l /(n+1)\right\}$ be a grid on the interval $(0,1)$ and $\bar{\omega}=\omega \cup\left\{\xi_{0}=0, \xi_{n+1}=l\right\}$ be a grid covering the closed interval $[0,1]$. Let $\mathbb{R}^{n}$ be the space of grid functions defined on $\bar{\omega}$ and vanishing at $\xi_{0}=0, \xi_{n+1}=l$. The matrix $B=B_{h}$ corresponding to the difference operator

$$
\begin{aligned}
D\left(B_{h}\right) & =\{y(x), x \in \bar{\omega}: y(0)=0, y(l)=0\}, \\
B y & =-y_{\bar{x} x} \forall y \in \mathbb{R}^{n}
\end{aligned}
$$

possesses eigenvalues $\lambda_{h, k}=\frac{4}{h^{2}} \sin ^{2} \frac{k \pi h}{2 l}$ corresponding to the eigenfunctions $y_{k}(x)=\sin \frac{k \pi x}{l}, x \in$ $\bar{\omega}, k=1,2, \ldots, n$ with the norm

$$
\left\|y_{k}(x)\right\|=\left(\sum_{j=1}^{n} h y_{k}^{2}\left(x_{j}\right)\right)^{1 / 2}=\sqrt{l / 2} .
$$

The vector $x_{0}=\sin \frac{m \pi x}{l}$ for each fixed $m$ is analytical for $B$ uniformly in $h$, since due to

$$
\begin{aligned}
B^{j} x_{0} & =\lambda_{h, m}^{j} \sin \frac{m \pi x}{l} \\
\left\|B^{j} x_{0}\right\| & =\lambda_{h, m}^{j} \sqrt{l / 2} \\
\left|\lambda_{h, m}\right| & \leqslant \frac{4}{h^{2}} \cdot \frac{2}{\pi} \cdot \frac{m \pi h}{2 l}=\left(\frac{2 m}{l}\right)^{2}
\end{aligned}
$$


we have that the series

$$
\sum_{j=0}^{\infty} \frac{\left\|B_{h}^{j} x_{0}\right\| t^{j}}{j !} \leqslant \sqrt{l / 2} \sum_{j=0}^{\infty}\left(\frac{2 m}{l}\right)^{2 j} \frac{t^{j}}{j !}
$$

converges for all $t \in(-\infty, \infty)$.

\section{Another form of representation and approximation II}

From (2.6) it follows that

$$
u_{p}=i B\left(u_{p}-u_{p-1}\right), p=0,1,2, \ldots ; u_{-1}=i B^{-1} x_{0} .
$$

Substituting this representation into (2.4) and using the summation by parts

$$
\sum_{n=1}^{N-1} u_{n} v_{n}=u_{N} v_{N-1}-u_{0} v_{0}-\sum_{n=0}^{N-1} u_{n} v_{n}
$$

we obtain

$$
\begin{aligned}
x(t) & =u_{0}+i B \sum_{n=1}^{\infty} L_{n}^{(0)}(t)\left(u_{n}-u_{n-1}\right)=u_{0}-i B u_{0} L_{0}^{(0)}(t)-i B \sum_{n=0}^{\infty}\left(L_{n+1}^{(0)}(t)-L_{n}^{(0)}(t)\right) u_{n} \\
& =x_{0}-i B \sum_{n=0}^{\infty}\left(L_{n+1}^{(0)}(t)-L_{n}^{(0)}(t)\right) u_{n} .
\end{aligned}
$$

Here we have used estimates (2.12) (which yield $\lim _{n \rightarrow \infty} L_{n}^{(0)}(t)=0$ for each fixed $t$ ) as well as $(2.11)$.

Remark 4.1. One can see that representation (4.3) satisfies Eq. (2.1) also using the well-known relation $[3,21]$

$$
\frac{d}{d t}\left[L_{n+1}^{(0)}(t)-L_{n}^{(0)}(t)\right]=-L_{n}^{(0)}(t)
$$

which, together with (2.4), yields

$$
\dot{x}(t)=i B \sum_{n=0}^{\infty} L_{n}^{(0)}(t) u_{n}=i B x(t) .
$$

It holds [21] that

$$
\left|L_{n}^{(0)}(t)-L_{n-1}^{(0)}(t)\right|=\frac{t}{n}\left|L_{n-1}^{(1)}(t)\right| \leqslant c t^{1 / 4} e^{t / 2} n^{-3 / 4}\left(1+n^{-1 / 4} t^{5 / 4}\right)
$$

uniformly in $t \in[0, T]$. Combining this estimate with (2.11), we obtain the following majorant for series $(4.3)$

$$
\sum_{p=1}^{\infty} p^{-\sigma / 2-3 / 4}
$$


which shows that the series converges provided that $\sigma>1 / 2$. For the truncated series

$$
x_{N}^{(1)}(t)=x_{N}^{(1)}\left(t ; x_{0}\right)=x_{0}-i B \sum_{n=0}^{N}\left(L_{n+1}^{(0)}(t)-L_{n}^{(0)}(t)\right) u_{n},
$$

which represents an algorithm for an approximate solution of problem (2.1), in view of (4.6) we have the following error estimate:

$$
\left\|x(t)-x_{N}^{(1)}(t)\right\| \leqslant c t^{1 / 4} e^{t / 2} N^{-\sigma / 2-1 / 4}\left\|B^{\sigma} x_{0}\right\| .
$$

In the case of an analytical initial vector $x_{0}$ we have instead of 3.14 the error estimate

$$
\left\|x(t)-x_{N}^{(1)}(t)\right\| \leqslant c t^{1 / 4} e^{t / 2} \sum_{p=N+1}^{\infty} p^{-3 / 4} e^{-c_{1} p^{1 / 3}}\left\|\left|x _ { 0 } \left\|\left|\leqslant c t^{1 / 4} e^{t / 2} N^{-1 / 12} e^{-c_{1} N^{1 / 3}}\left\|\mid x_{0}\right\| .\right.\right.\right.\right.
$$

Estimates $(4.9),(4.10)$ indicate that the approximation error $x_{N}^{(1)}(t)$ tends to zero as $N \rightarrow \infty$ but also together with $t \rightarrow 0$.

Note that we can rewrite representation (4.3) and algorithm (4.8) in the form

$$
\begin{aligned}
x(t) & =u_{0}+i B \sum_{n=1}^{\infty} L_{n}^{(0)}(t)\left(u_{n}-u_{n-1}\right)=u_{0}+i B \sum_{n=1}^{\infty} L_{n}^{(0)}(t)\left(u_{n}-u_{n-1}\right) \\
& =x_{0}-\sum_{n=0}^{\infty}\left(L_{n+1}^{(0)}(t)-L_{n}^{(0)}(t)\right) \tilde{u}_{n}, \\
x_{N}^{(1)}(t) & =x_{0}-\sum_{n=0}^{N}\left(L_{n+1}^{(0)}(t)-L_{n}^{(0)}(t)\right) \tilde{u}_{n},
\end{aligned}
$$

where (cf. (2.6))

$$
\begin{aligned}
(i B-I) \tilde{u}_{0} & =-i B x_{0}, \\
(i B-I) \tilde{u}_{p+1} & =i B \tilde{u}_{p}, \quad p=0,1, \ldots
\end{aligned}
$$

From (4.12) we have

$$
\begin{aligned}
\tilde{u}_{0} & =-T x_{0}, \\
\tilde{u}_{p+1} & =T \tilde{u}_{p}=T^{p+2} x_{0}, \quad p=0,1, \ldots
\end{aligned}
$$

The equivalent form of (4.11)) is

$$
\begin{gathered}
x(t)=e^{i B} x_{0}, e^{i B}=I-\sum_{n=0}^{\infty}\left(L_{n+1}^{(0)}(t)-L_{n}^{(0)}(t)\right) T^{n+1}, \\
x_{N}^{(1)}(t)=e_{N}^{i B} x_{0}, e_{N}^{i B}=I-\sum_{n=0}^{N}\left(L_{n+1}^{(0)}(t)-L_{n}^{(0)}(t)\right) T^{n+1} .
\end{gathered}
$$

Given the solution operator $S(t)=S(t ; B)=e^{i B t}$ for the homogeneous Schrödinger equation (2.1), the solution of the inhomogeneous Schrödinger equation

$$
\dot{x}(t)=i B x(t)+f(t), \quad x(0)=x_{0} .
$$


with a given function $f(t)$ can be represented by

$$
x(t)=e^{i B t} x_{0}+\int_{0}^{t} e^{i B(t-\tau)} f(\tau) d \tau .
$$

Example 4.1. The problem

$$
\dot{u}(t)=i B u(t)+f(t), \quad u(0)=u_{0} .
$$

with

$$
\begin{aligned}
D(B) & =\left\{u(x) \in H^{2}(-\infty, \infty): \lim _{x \rightarrow \pm \infty} u(x)=0\right\}, \\
B u & =-\frac{d^{2} u}{d x^{2}} \\
f(t) & =f(t, x)=e^{-x^{2}}\left(1-2 i t+4 i t x^{2}\right), u_{0}=u_{0}(x)=e^{-x^{2}}
\end{aligned}
$$

possesses the exact solution $u(t, x)=t e^{-x^{2}}$. Using the well known formula [3] (here $L_{n}^{(0)}(t)=$ $\left.L_{n}(t)\right)$

$$
\frac{d}{d t}\left[L_{n}(t)-L_{n+1}(t)\right]=L_{n}(t)
$$

as well as the intgration by parts one obtains easily

$$
\int_{0}^{t} \tau L_{n}(\tau) d \tau=(t-1)\left(L_{n}(t)-L_{n+1}(t)\right)+\left(L_{n+1}(t)-L_{n+2}(t)\right)
$$

$\int_{0}^{t} \tau^{2} L_{n}(\tau) d \tau=\left(t^{2}-2 t+2\right)\left(L_{n}(t)-L_{n+1}(t)\right)+(2 t-1)\left(L_{n+1}(t)-L_{n+2}(t)\right)+\left(L_{n+2}(t)-L_{n+3}(t)\right)$ and further

$$
\int_{0}^{t} \tau L_{n}(t-\tau) d \tau=-\left(L_{n}(t)-L_{n+1}(t)\right)+\left(L_{n+1}(t)-L_{n+2}(t)\right)
$$

$\int_{0}^{t} \tau^{2} L_{n}(t-\tau) d \tau=2(t+1)\left(L_{n}(t)-L_{n+1}(t)\right)+(2 t-1)\left(L_{n+1}(t)-L_{n+2}(t)\right)+\left(L_{n+2}(t)-L_{n+3}(t)\right)$.

These formulas together with (2.4) imply the following representation for the solution of (4.17), (4.18)

$$
\begin{aligned}
& u(t, x)=\int_{0}^{t} e^{i B(t-\tau)}\left[e^{-x^{2}}\left(1-2 i \tau+4 i \tau x^{2}\right)\right] d \tau \\
& =\sum_{n=0}^{\infty}\left\{\int_{0}^{t} L_{n}^{(0)}(t-\tau)\left[e^{-x^{2}}\left(1-2 i \tau+4 i \tau x^{2}\right)\right] d \tau\right\} u_{n} \\
& =e^{-x^{2}} \sum_{n=0}^{\infty}\left\{-\left(L_{n}(t)-L_{n+1}(t)\right)\right\} u_{n}-2 i e^{-x^{2}} \sum_{n=0}^{\infty}\left\{-\left(L_{n}(t)-L_{n+1}(t)\right)+\left(L_{n+1}(t)-L_{n+2}(t)\right)\right\} u_{n} \\
& +4 i x^{2} e^{-x^{2}} \sum_{n=0}^{\infty}\left\{2(t+1)\left(L_{n}(t)-L_{n+1}(t)\right)+(2 t-1)\left(L_{n+1}(t)-L_{n+2}(t)\right)+\left(L_{n+2}(t)-L_{n+3}(t)\right)\right\} u_{n} .
\end{aligned}
$$




\section{Preconditioning}

In the case of a bounded self-adjoint operator $B$ with the spectrum in $\left[\lambda_{0}, \Lambda_{0}\right], \lambda_{0}>0$, we have $u_{p+1}=\int_{\lambda_{0}}^{\Lambda_{0}}\left(\frac{i \lambda}{\lambda+i}\right)^{p} d E_{\lambda} x_{0}$, where $E_{\lambda}$ is the spectral family of $B$. Taking into account that

$$
\begin{aligned}
\left|\frac{i \lambda}{\lambda+i}\right| & =\left|\frac{\lambda(\lambda-i)}{\lambda^{2}+1}\right|=\frac{\sqrt{\lambda^{4}+\lambda^{2}}}{\lambda^{2}+1} \\
& =\frac{\sqrt{\left(\lambda^{2}+1\right)^{2}-\lambda^{2}-1}}{\lambda^{2}+1}=\sqrt{1-\frac{1}{\lambda^{2}+1}} \leqslant \sqrt{1-\frac{1}{\Lambda_{0}^{2}+1}} \equiv q \equiv q_{B_{h}}
\end{aligned}
$$

this representation yields the estimate

$$
\left\|u_{p+1}\right\| \leqslant q^{p}\left\|x_{0}\right\|
$$

Estimate (3.3) can be modified now to

$$
\begin{aligned}
\left\|x(t)-x_{N}(t)\right\| \leqslant c\left\|x_{0}\right\| \begin{cases}t^{-\frac{1}{4}} e^{\frac{t}{2}} \sum_{p=N+1}^{\infty} p^{-1 / 4} q^{p}, & \text { if } t \in[a, b] \\
e^{\frac{t}{2}} \sum_{p=N+1}^{\infty} q^{p}, & \text { if } t \in[0, b]\end{cases} \\
\leqslant c\left\|x_{0}\right\| \begin{cases}t^{-\frac{1}{4}} e^{\frac{t}{2}}(N+1)^{-1 / 4} \frac{q^{N+1}}{1-q}, & \text { if } t \in[a, b] \\
e^{\frac{t}{2}} \frac{q^{N+1}}{1-q}, & \text { if } t \in[0, b] .\end{cases}
\end{aligned}
$$

If $B=B_{h}$ is a finite difference or the FEM approximation of a second-order elliptic operator, then $\Lambda_{0}=c / h^{2}$, where $c$ is a constant and $h \rightarrow 0$ is the characteristic mesh parameter. In this case, we have

$$
q=\sqrt{c^{2} /\left(c^{2}+h^{4}\right)} \rightarrow 1
$$

as $h \rightarrow 0$, i.e., the convergence of series (2.3) or (2.3) can be slow.

To overcome this difficulty, one can use the following simple preconditioning of the matrix $B_{h}$. Let $\alpha=2^{M}$ be such that $\Lambda_{0} \leqslant \alpha$. We consider the matrix $B_{h, \alpha}=B_{h} / \alpha$ and the operator exponential $E_{\alpha}=e^{i B_{h, \alpha} t}$. The matrix $E_{\alpha}$ can be computed by algorithm (3.1) which converges at least exponentially as a geometric progression with a denominator $q_{B_{h, \alpha}}$. Since the spectrum of $B_{h, \alpha}$ lies now in $\left(0, \Lambda_{0} / \alpha\right)$ and this interval can be made arbitrarily small, then due to (5.1) we can make $q_{B_{h, \alpha}}$ arbitrarily small and arrive at a very rapid convergence. The required solution matrix $e^{i B_{h} t}$ can be computed by the following squaring algorithm in $M$ steps:

\section{Algorithm ES.}

This algorithm computes $e^{i B_{h} t}$ from $E_{\alpha}=e^{i B_{h, \alpha} t}$.

Input: $t, E_{\alpha}=e^{i B_{h, \alpha} t}$

Output: $E=e^{i B_{h} t}$

1. Set $E:=E_{\alpha}$.

2. For $k=1: M$ do $E:=E * E$.

A similar so-called Scaling and Squaring Method was described in [19], p.241, but to compute the matrix exponential, the Taylor or Padé expansions of $e^{z}$ were used. 
Indeed, this algorithm cannot be used in the case of an unbounded $B$. In this case, one can make use of algorithm $(4.8)$ and estimates $(4.9,4.10)$ which indicate the convergence of the algorithm with respect to $N \rightarrow \infty$ or $t \rightarrow 0$. Suppose we can compute $x\left(t_{0}\right)$ efficiently for some $t_{0}$ though we are to compute $x(t)$ for $t=2^{M} t_{0}$. The following algorithm does it in $M$ steps.

\section{Algorithm ES1 .}

This algorithm computes $e^{i B t}$ for $t=2^{M} t_{0}$ using (4.8).

Input: $x_{0}, t_{0}, M, N$

Output: $x_{N}^{(1)}(t)$

1. Set $X_{0}:=x_{0}$ and compute $X:=x_{N}^{(1)}\left(t_{0} ; X_{0}\right)$ by (4.8).

2. For $k=1: M$ do

\section{begin}

2.1. $X_{0}:=X$

2.2. Compute $X:=x_{N}^{(1)}\left(t_{0} ; X_{0}\right)$ by $(4.8)$

\section{end}

This algorithm exploits the relation $e^{i B 2 t_{0}}=\left(e^{i B t_{0}}\right)^{2}$ and is also similar to the Scaling and Squaring Method.

Remark 5.1. Using the well-known correspondence for the Laplace transform

$$
\mathcal{L}\left\{t^{n-1} e^{a t}\right\}=\frac{\Gamma(n)}{(s-a)^{n}},
$$

as well as the correspondence

$$
\mathcal{L}\left\{t^{\alpha} L_{n}^{(\alpha)}(t)\right\}=\frac{\Gamma(\alpha+n+1)(s-1)^{n}}{n ! s^{\alpha+n+1}}, \Re \alpha>-1, \Re s>0,
$$

which for $\alpha=0$ reads as

$$
\mathcal{L}\left\{L_{n}^{(0)}(t)\right\}=\frac{(s-1)^{n}}{s^{n+1}},
$$

we obtain for $(2.3)$

$$
\mathcal{L}\left\{e^{i B t} x_{0}\right\}=\mathcal{L}\left\{\sum_{n=0}^{\infty} L_{n}^{(0)}(t) u_{n}\right\}=\mathcal{L}\left\{\sum_{n=0}^{\infty} L_{n}^{(0)}(t) u_{n}\right\}=\Sigma(s)
$$

where

$$
\Sigma(s)=\sum_{n=0}^{\infty} \frac{(s-1)^{n}}{s^{n+1}}\left[i B(i B-I)^{-1}\right]^{p-1} u_{0}=\frac{s-1}{s^{2}}\left[I-\frac{s-1}{s}\left(i B(i B-I)^{-1}\right)\right]^{-1} u_{0}
$$

and $u_{0}=-(i B-I)^{-1} x_{0}$. Note that here thought was given to the fact that $\left\|i B(i B-I)^{-1}\right\| \leqslant$ 1 and $\left|\frac{s-1}{s}\right|<1$ for $\Re s>1 / 2$, as well as the formula for the sum of infinite geometric series, has been used. Thus, the discrete spectrum of the Schrödinger operator coincides with the poles of the function $\|\Sigma(s)\|$ multiplied by $-i$. 


\section{References}

1. N.I. Achieser and I.M. Glasmann, Theorie der linearen operatoren im Hilbert-Raum, AkademieVerlag, Berlin, 1975.

2. D.Z. Arov, I.P. Gavrilyuk, and V.L. Makarov, Representation and approximation of the solution of an initial value problem for a first order differential equation with an unbounded operator coefficient in Hilbert space based on the Cayley transform, In C. Bandle et al., editor, Progress in partial differential equations, Pitman Res. Notes Math. Sci., 1 (1994), pp. 40-50.

3. H. Bateman and A. Erdelyi, Higher Transcendental Functions, v. 2, MC Graw-Hill Book Comp., Inc., New York,Toronto, London, 1988. 1991.

4. I. N. Bronstein and K.A. Semendjajew, Taschenbuch der Mathematik, Teubner, Stuttgart, Leipzig,

5. D. Z. Arov and I. P. Gavrilyuk, A method for solving initial value problems for linear differential equations in hilbert space based on the cayley transform, Numer. Func. Anal. and Optimiz., 14 (1993), pp. 459-473.

6. I. P. Gavrilyuk, Strongly P-positive operators and explicit representation of the solutions of initial value problems for second order differential equations in Banach space, Journ.of Math. Analysis and Appl., 236 (1999), pp. 327-349.

7. I. P. Gavrilyuk, W. Hackbusch, and B. N. Khoromskij, $\mathcal{H}$-matrix approximation for the operator exponential with applications, Numer. Math., 92 (2002), pp. 83-111.

8. I. P. Gavrilyuk, W. Hackbusch, and B. N. Khoromskij, Data- sparse approximation to the operatorvalued functions of elliptic operator, Math. Comp., 73 (2004), pp. 1297-1324.

9. I. P. Gavrilyuk, W. Hackbusch, and B. N. Khoromskij, Tensor-product approximation to elliptic and parabolic solution operators in higher dimensions, Computing, 74 (2005), pp. 131-157.

10. I. P. Gavrilyuk and V. L. Makarov, The Cayley transform and the solution of an initial value problem for a first order differential equation with an unbounded operator coefficient in Hilbert space, Numer. Func. Anal. Optimiz., 15 (1994), pp. 583-598.

11. I. P. Gavrilyuk and V. L. Makarov, Representation and approximation of the solution of an initial value problem for a first order differential eqation in Banach space, Z. Anal. Anwend.(ZAA), 15 (1996), pp. $495-527$.

12. I. P. Gavrilyuk and V. L. Makarov, Explicit and approximate solutions of second order elliptic differential equations in Hilbert- and Banach spaces, Numer. Func. Anal. Optimiz., 20 (1999), no. 7\&8, pp. 695-717.

13. I. P. Gavrilyuk and V. L. Makarov, Explicit and approximate solutions of second order evolution differential equations in Hilbert space, Numerical Methods for Partial Differential Equations, 15 (1999), pp. 111-131.

14. I. P. Gavrilyuk and V. L. Makarov, Strongly positive operators and numerical algorithms without accuracy saturation (in Russian), Publishing House of the Institute of Mathematics of the Academie of Sciences of Ukraine, Kiev, 2004.

15. I. P. Gavrilyuk and V. L. Makarov, Algorithms without accuracy saturation for evolution equations in Hilbert and Banach spaces, Math. Comp., 74 (2005), pp. 555-583.

16. I. P. Gavrilyuk and V. L. Makarov, An explicit boundary integral representation of the solution of the two-dimensional heat equation and its discretization, J. Integral Equations Appl., 12 (2000), no. 1, pp. 63-83.

17. I. P. Gavrilyuk, An algorithmic representation of fractional powers of positive operators, Numerical Funct. Anal. Optimization, 17 (1996), no. 3-4, pp. 293-305.

18. J. A. Goldstein, Semigroups of Linear Operators and Applications, Oxford University Press and Clarendon Press, New York, Oxford, 1985.

19. I. Higham, Functions of Matrices. Theory and Computation, SIAM, Manchester, 2008.

20. M. G. Krein, On fundamental approximating problem in the extrapolation theory and filtration of stationary stochastic processes (in Russian), Doklady AN SSSR, 94 (1954), no. 1, pp. 13-16.

21. P. K. Suetin, Classical Orthogonal Polynomials (in Russian), Naukova Dumka, Moscow, 1979.

22. G. Szegö, Orthogonal Polynomials, American Mathematical Society, New York, 1959. 